\title{
INDUCTION OF PSEUDOPREGNANCY IN THE GOLDEN HAMSTER
}

\author{
MILTON DIAMOND AND RYUZO YANAGIMACHI \\ Department of Anatomy, Universitv of Hawaii School of Medicine, \\ Honolulu, Hawaii 96822
}

(Received 1st March 1968)

In the hamster, unlike the rat in which pseudopregnancy is induced with ease, past attempts to induce pseudopregnancy by mechanical vaginal-cervical stimulation have been unsuccessful (Carlson \& DeFeo, 1953; DeFeo, 1966.) Pseudopregnancy in this species is usually induced by mating an oestrous female with a vasectomized buck overnight. The wide use of the hamster, however, in ovarian and reproductive studies indicated to us that an alternative means of inducing pseudopregnancy would prove of practical experimental value. The present study reports various attempts to induce pseudopregnancy mechanically and compares the results with those obtained by sterile matings.

Mature virgin females, 3 to 6 months old, weighing 100 to $160 \mathrm{~g}$ were used. They were housed in groups in plastic cages $(16 \times 12 \times 6$ in.) and placed singly in cages for one oestrous cycle before stimulation or mating. Stimulation of oestrous females was achieved by vaginal insertion of a mechanical vibrator (Vibro-Graver, Burgess Vibro Crafters Inc., Chicago). Mating was achieved by placing an oestrous female with a vasectomized buck in an observation cage $(16 \times 18 \times 9$ in. $)$. Females were judged in oestrus if they had transparent vaginal mucus (Orsini, 1961) and showed a lordosis response to the experimenter stroking her back, as well as to vaginal inspection by the male hamster. Mechanical stimulation was done either by one continuous vibration for $150 \mathrm{sec}$ or by five, fifteen or thirty 5-sec vibrations evenly spaced over 150, 300 or $600 \mathrm{sec}$. The stimulating probe ( $4 \mathrm{~mm}$ diameter) was inserted into the cervix while the female was held on a flat surface. For mating, each female was placed with a vasectomized buck for comparable periods of 150,300 or 600 sec.

Pseudopregnancy was evaluated in every case by daily vaginal checks (Orsini, 1961). Occasionally confirmation was sought in all groups by: (a) the traumatic induction and maintenance of a decidual reaction (DeFeo, 1967); (b) histological examination of the ovary; or (c) the bearing of young by females artificially inseminated. After mating or mechanical stimulation (Day 0 ), all animals were checked for a minimum of 10 days for possible delayed pseudopregnancy, i.e. the onset of pseudopregnancy in the following cycle. No instances of delayed pseudopregnancy were found.

Table 1 shows that exposure of an oestrous female to a vasectomized buck, even for $150 \mathrm{sec}$, was sufficient to induce pseudopregnancy (40\%). Leaving the female with the male for 300 or $600 \mathrm{sec}$ increased the success rates significantly, 90 and $80 \%$, respectively. The efficacy of these short durations is notable when 
compared with the findings of Beach \& Rabedeau (1959) who showed that the intact male hamster, allowed to copulate freely, reaches exhaustion in approximately $900 \mathrm{sec}$ (within this time there were, on average, nine ejaculations and thirty-two intromissions) and then goes into a fairly protracted rest period; and with the findings of Greenwald \& Peppler (personal communication) that, of oestrous females placed with males overnight, only $81.5 \%$ ( 137 of 168 ) actually become pregnant. Beach \& Rabedeau (1959) did not check for pseudopregnancy.

Mechanical stimulation met with variable degrees of success in inducing pseudopregnancy. This success appeared to depend upon the combined effect of the number of separate and discrete stimuli applied and the total time-span over which the stimuli were administered.

Only $20 \%$ of the females stimulated with a single, continuous $150-\mathrm{sec}$ vibration became pseudopregnant; with five stimuli, each of $5 \mathrm{sec}$ duration, the response was not improved. Of the females receiving fifteen or more 5-sec

TABLE 1

INDUCTION OF PSEUDOPREGNANCY IN THE HAMSTER

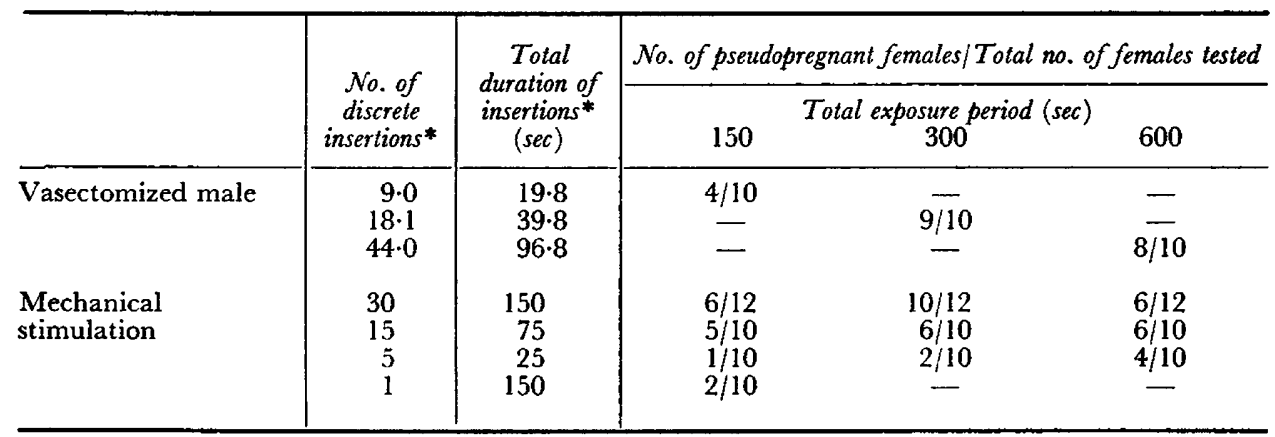

* Values for vasectomized male tests represent means.

vibrations, however, at least half became pseudopregnant. The most successful mechanical procedure for inducing pseudopregnancy $(83 \%)$ was to stimulate the female thirty times over $300 \mathrm{sec}$. Fewer insertions, or lengthening or shortening the exposure duration markedly decreased success. Stimulation for a much shorter interval than $150 \mathrm{sec}$ and with a single insertion most probably accounted for the lack of success previously reported for this species (Carlson \& DeFeo, 1963; DeFeo, personal communication 1968).

While the number of insertions is directly related to the total time of vaginalcervical stimulation (no. of insertions $\times$ duration of each insertion), neither this total time alone, nor the absolute number of insertions are independently crucial in inducing pseudopregnancy. That the absolute number of stimuli, over a minimum, is not crucial is obvious from similar success rates in groups of females mechanically stimulated fifteen or thirty times over $150-$ or 600 -sec periods and from the mating tests where the males averaged nine, eighteen and forty-four insertions (intromissions and ejaculations) for 150,300 and $600 \mathrm{sec}$, respectively, with the latter two groups being equally successful in inducing 
pseudopregnancy. This finding is comparable to results reported for the rat (Wilson, Adler \& Le Boeuf, 1965). Further, no significant differences existed between the number of insertions received by the females that did, or did not, become pseudopregnant. The total duration of one insertion is not crucial since, of those females stimulated continuously for $150 \mathrm{sec}$, which was the maximum time of total insertion for any group, only $20 \%$ became pseudopregnant. Further, the mechanically-stimulated females were generally exposed to longer total times of vaginal-cervical stimulation than mated females but proportionately fewer became pseudopregnant (Table 1). Considering a mean penile intromission duration of $2.2 \mathrm{sec}$, as judged from kymograph records we made during visual observation of the mating sequence, the mean total intromission times become approximately 20, 40 and $100 \mathrm{sec}$ for the three mated groups in contrast with the 25-, 75- and 150-sec periods of mechanical stimulation. The successful induction of pseudopregnancy among the mated females was independent of the vasectomized males having ejaculated or not.

A typical decidual response reaction was invariably seen after cutting the entire length of the antemesometrial wall of the uterus 5 days after mechanical stimulation and examining the uterus 4 days later (DeFeo, 1967). On Day 5 of pseudopregnancy the ovaries invariably contained large, apparently active corpora lutea. In females artificially inseminated with spermatozoa placed in the uterine horns within $30 \mathrm{~min}$ after mechanical stimulation (thirty 5-sec vibrations in $300 \mathrm{sec}$ ), healthy and viable young were delivered on the 16th days after insemination. The mothers nursed successfully.

While it has now been demonstrated that the induction of pseudopregnancy by sterile mating or mechanical stimulation is possible and convenient in the hamster, much remains to be understood about the processes involved. Since pseudopregnancy occurred more frequently and more reliably after mating than with mechanical stimulation despite fewer and shorter stimuli, some crucial feature of the mating situation is still absent or inconsistently controlled during artificial stimulation. This has been intimated before by comparable findings in the rat (Haterius, 1933). In any case, the need for multiple and distinct vaginal stimuli to be present, along with a number of other conditions has been manifest.

Appreciation for co-operation in the execution of this research is extended to Miss Judy Carsello and Mrs Elizabeth Obata. Dr Vincent DeFeo assisted with testing of the decidual reactions. This research was supported in part by Public Health Service Grants HD 03394 and HD 02066 of the National Institute of Child Health and Human Development of the National Institutes of Health and in part by a grant from the Ford Foundation.

\section{REFERENCES}

Beach, F. A. \& Rabedeau, R. G. (1959) Sexual exhaustion and recovery in the male hamster. $\mathcal{F}$. comp. physiol. Psychol. 52, 56.

Carlson, R. R. \& DeFEo, V.J. (1963) Comparative effectiveness of physiological, mechanical, and pharmacological means of inducing pseudopregnancy in the rat, mouse, and hamster. Anat. Rec. 145, 312. 
DEFEO, V. J. (1966) Vaginal-cervical vibration: a simple and effective method for the induction of pseudopregnancy in the rat. Endocrinology, 79, 440.

DeFeo, V. J. (1967) Decidualization. In: Cellular Biology of the Uterus, p. 252. Ed. R. M. Wynn. Appleton Century Crofts, New York.

Haterius, H. O. (1933) Partial sympathectomy and induction of pseudopregnancy. Am. F. Physiol. 103, 97.

Orsini, M. W. (1961) The external vaginal phenomena characterizing the stages of the estrous cycle, pregnancy, pseudopregnancy, lactation, and the anestrous hamster, Mesocricetts auratus Waterhouse. Proc. Anim. Care Panel, 11, 193.

Wirson, J. R., Adler, N. \& Le Boeuf, B. (1965) The effects of intromission frequency on successful pregnancy in the female rat. Proc. natn. Acad. Sci. U.S.A. 53, 1392. 